\title{
Lateral Spin Diffusion Probed by Two-Color Hanle-MOKE Technique
}

\author{
J.-H. Quast*, G.V. Astakhov, W. Ossau, L.W. MolenkamP \\ Physikalisches Institut (EP3), Universität Würzburg, 97074 Würzburg, Germany
}

\section{J. Heinrich, S. Höfling And A. Forchel}

Physikalisches Institut (TP), Universität Würzburg, 97074 Würzburg, Germany

We report on all optical spatially resolved spin diffusion experiments in an unstrained, unbiased $n$-GaAs layer. Optical pump and probe intensities are varied over a wide range to study the impact of optical disturbance on spin transport. Both quantities have a considerable influence on the measured spin diffusion length and spin lifetime. Furthermore, an effective spin diffusion coefficient was obtained as a function of temperature.

PACS numbers: 72.25.Dc, 72.25.Fe, 85.75.-d, 72.25.Rb

\section{Introduction}

In order to use spin degrees of freedom as information carriers it is necessary to control, manipulate and monitor spins. Optical selection rules provide powerful tools to create and probe electron spin polarization in semiconductors [1]. Microscopic spin diffusion over tens of microns was observed in $n$-GaAs using the Hanle effect [2] and it has been demonstrated that electrical spin drag over hundreds of microns is possible [3-5]. The magneto-optical Kerr effect (MOKE) is used in a number of studies to record lateral images of diffusing and drifting spins [6-8]. In order to obtain reasonable signal to noise ratios it is customary to tune the probe laser energy close to a resonance. As a by-product this results in the excitation of additional electron-hole pairs by the probe laser. A common strategy to avoid this influence is to reduce the laser power to the lowest possible value. The data presented here analyze the residual disturbance caused by the probe laser. Further, the intensity of the pump light has a well known influence on the spin polarization and spin lifetime [1], but the effect of strong pumping on lateral spin diffusion has not been investigated so far.

*corresponding author; e-mail: jquast@physik.uni-wuerzburg.de 


\section{Experimental setup}

The presented data were obtained for a Si-doped (nominally $n_{\mathrm{e}}=$ $\left.2.5 \times 10^{16} \mathrm{~cm}^{-3}\right) n$-type GaAs layer of $1.5 \mu \mathrm{m}$ thickness. It was grown by molecular beam epitaxy (MBE) on a semi-insulating (001) GaAs substrate. Between substrate and the top layer a $200 \mathrm{~nm}$ buffer layer (undoped GaAs), followed by a $5 \mathrm{~nm}$ barrier (AlAs) and a $100 \mathrm{~nm}$ spacer layer (undoped GaAs) have been grown. The sample is mounted strain-free inside a helium-flow cryostat and kept at a temperature $T=8 \mathrm{~K}$. A two-color Kerr rotation technique is used for optical spin injection and detection [9]. Optical spin pumping is performed by a solid state laser $(785 \mathrm{~nm})$ which is modulated between $\sigma^{+}$and $\sigma^{-}$polarization with a frequency of $50 \mathrm{kHz}$ by a photoelastic modulator (PEM). The net spin polarization perpendicular to the sample surface is probed using the magneto-optical Kerr effect $[6,9]$. For this purpose the Kerr rotation angle $\theta$ introduced to the beam of a constant-wave titanium-sapphire laser upon reflection from the sample surface is measured by balanced photodiodes and demodulated by a lock-in amplifier. The setup used for scanning Kerr microscopy is shown in Fig. 1a. Surface scans in the $x-y$ plane are performed using a microscope objective (numerical aperture 0.14) mounted on a NanoCube ${ }^{\mathrm{TM}}$ piezo system. Figure $1 \mathrm{~b}$ shows a knife-edge scan of the probe laser focus. Its derivative (inset) yields the spot profile, which is well described by a Gaussian with a half-width-at-half-maximum (HWHM) of $\Delta_{\text {probe }}=2.7 \mu \mathrm{m}$. The pump beam is directed under $45^{\circ}$ angle of incidence on the sample surface and is focused by a second objective of the same design as the one used for the probe beam. Inside the sample electron spins are optically oriented under an angle of $\gamma=11^{\circ}$ with respect to the sample normal (direction of the probe beam according to the Snell law). The data presented below have been obtained by scanning along $x$ direction, assuming isotropic behavior of the sample. For the Hanle effect studies an external magnetic field is also applied along $x$ direction.

\section{Sample characterization}

First, we characterize the sample by polarization resolved photoluminescence (PL) and two-color Hanle-MOKE in the non-diffusive regime (laser spots are much larger than the typical spin diffusion length) by defocusing the beams to spot sizes of $c a .100 \mu \mathrm{m}$. The PL spectrum in Fig. 1c shows two lines assigned to the donoracceptor transition $\left(\mathrm{D}^{0}, \mathrm{~A}^{0}\right)$ and the donor-bound exciton $\left(\mathrm{D}^{0}, \mathrm{X}\right)$. In the same figure the degree of optically induced circular polarization $\rho_{\mathrm{c}}=\left(I_{-}-I_{+}\right) /\left(I_{-}+I_{+}\right)$ ( $I_{ \pm}$is the PL intensity of the $\left(\mathrm{D}^{0}, \mathrm{X}\right)$-line in the $\sigma^{ \pm}$polarization) is shown as a function of the excitation energy. A maximum polarization of $22 \%$ (theoretical limit is $25 \%$ [1]) is achieved for quasi-resonant excitation. The photon energy used to pump electron spins in the following experiments is fixed to $E_{\text {pump }}=1.579 \mathrm{eV}$ $(785 \mathrm{~nm})$. The optically induced Kerr rotation as a function of the probe energy is 

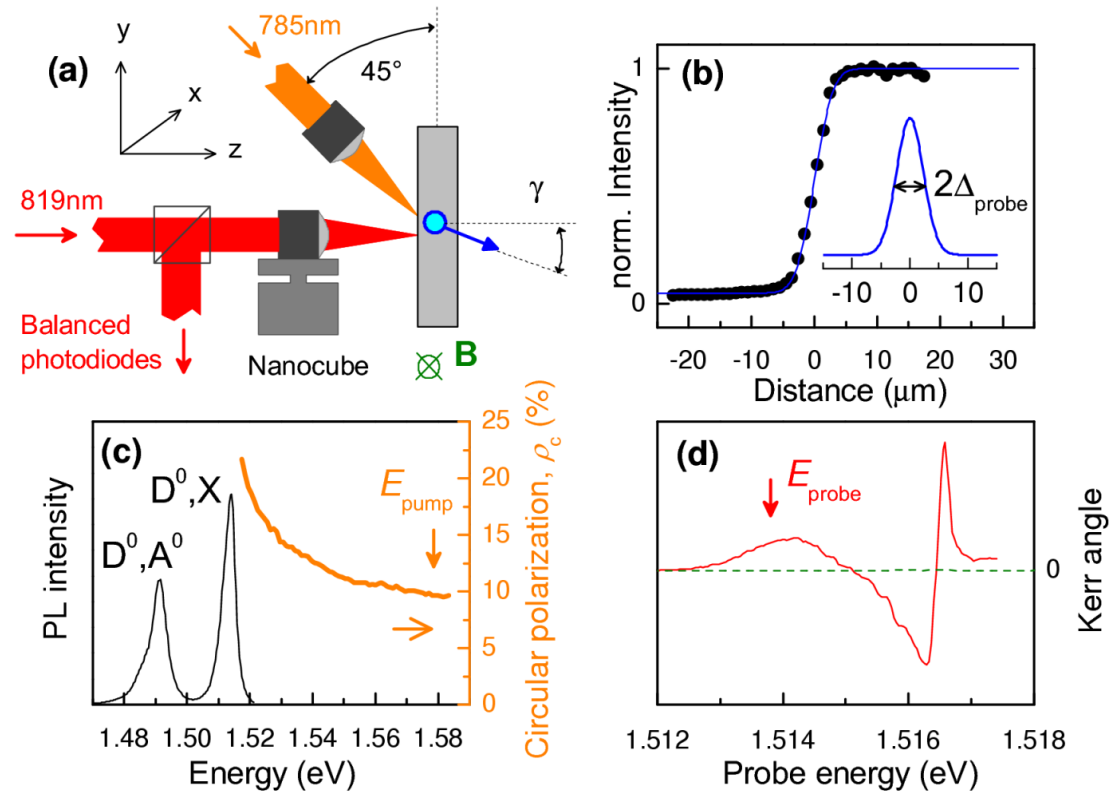

Fig. 1. Setup and sample characterization in non-diffusive regime. (a) Two-color Kerr microscopy setup. (b) Knife edge scan of the probe laser spot. Inset: laser spot profile with $\Delta_{\text {probe }}=2.7 \mu \mathrm{m}$. (c) PL and polarization excitation spectra. (d) Kerr angle $\theta$ vs. probe energy. The arrow indicates the probe energy $E_{\text {probe }}=1.514 \mathrm{eV}$ set in the following experiments. Pump energy is set to $E_{\mathrm{pump}}=1.579 \mathrm{eV}$.

shown in Fig. 1d. The probe energy is fixed to $E_{\text {probe }}=1.514 \mathrm{eV}(819 \mathrm{~nm})$ for the following experiments, which corresponds to the donor-bound exciton transition.

\section{Power dependence of spin diffusion}

Lateral scans of the steady state spin diffusion profile are shown in Fig. 2a for different pump intensities. The dotted line shows the excitation profile as detected by the probe spot. It corresponds to the convolution of pump and probe spots and yields a net resolution of $\Delta=7.5 \mu \mathrm{m}$ (HWHM) of our setup. A gradual increase in the spatial HWHM $\left(X_{1 / 2}\right)$ with pump intensity is clearly seen. This effect is summarized for different probe intensities in Fig. 2b. As a general trend the spin diffusion length decreases with increasing probe intensity, as seen from Fig. 2c. In order to examine the origin of such a behavior, we plot in Fig. $3 a$ and $b$ the Kerr rotation $\theta$ at the excitation point (i.e., at $x=0$ ) as a function of pump and probe intensities, respectively. For the pump intensity this behavior is fitted well by [1]:

$$
\theta\left(I_{\text {pump }}\right)=\frac{\theta_{\mathrm{S}}}{1+I_{\mathrm{S}} / I_{\text {pump }}},
$$

where $\theta_{\mathrm{S}}$ is the Kerr angle in saturation and $I_{\mathrm{S}}$ characterizes the intensity required 

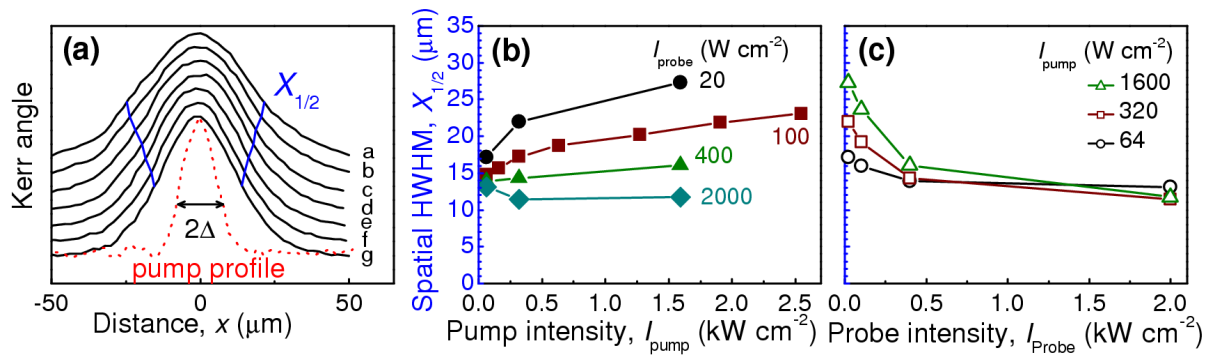

Fig. 2. Power dependences of the spin diffusion profile. (a) Spatial spin distribution for different pump intensities (from (a) to $(\mathrm{g}$ ): 2.5, 1.9, 1.3, 0.64, 0.32, 0.16, and $0.064 \mathrm{~kW} \mathrm{~cm}^{-2}$ ) obtained with a probe intensity of $0.1 \mathrm{~kW} \mathrm{~cm}^{-2}$ at a temperature $T=8 \mathrm{~K}$. Half width of these curves is marked by $X_{1 / 2}$. The dotted line represents the convolution of pump and probe laser spots (net resolution). The curves are shifted in constant steps for clarity. (b) $X_{1 / 2}$ vs. pump intensity for several probe intensities. (c) $X_{1 / 2}$ vs. probe intensity for several pump intensities. Lines in (b) and (c) are guides to the eye.
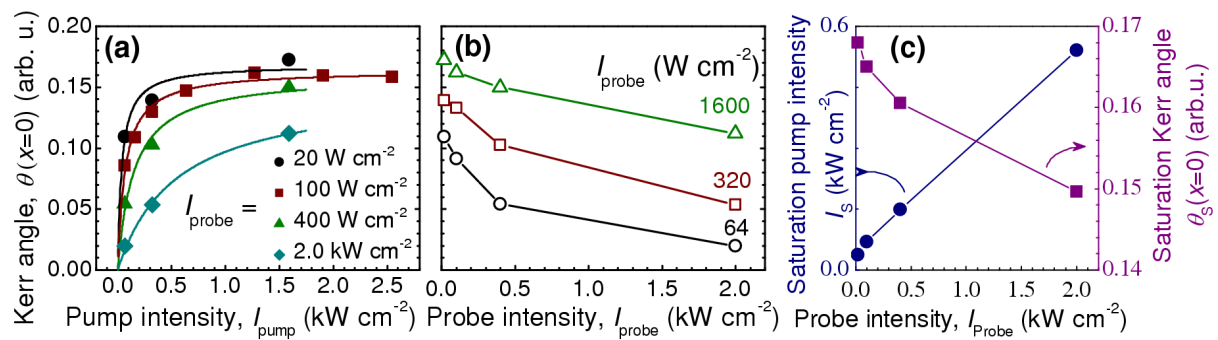

Fig. 3. Power dependences of the Kerr angle at $x=0$. (a) Kerr angle at the center of the pump spot vs. pump intensity for several probe intensities. Lines are fits to Eq. (1). (b) Kerr angle at the center of the pump spot vs. probe intensity for several pump intensities. Lines are guides to the eye. (c) Parameters $\theta_{\mathrm{S}}$ (right axis) and $I_{\mathrm{S}}$ (left axis) obtained from the fit to Eq. (1) of curves in part (a) vs. probe intensity.

to achieve the saturation. From the curves in Fig. 3a it is already clear that $I_{\mathrm{S}}$ grows with increasing probe intensity, whereas $\theta_{\mathrm{S}}$ is nearly independent of the latter. These relations are shown explicitly in Fig. 3c. Generally, the characteristic intensity $I_{\mathrm{S}}$ depends on the ratio of spin generation and decay rates. The faster the spins decay, the stronger one has to pump to go into saturation. The probe light hence generates holes, the recombination of which with spin polarized electrons introduces an additional spin decay channel. The efficiency of this channel scales with the ratio of the spin relaxation time and electron-hole recombination time, and hence may be effective even for moderate intensities. As a consequence, the spin polarization is destroyed and the spin diffusion is suppressed for increasing probe intensity as shown in Fig. 3b and Fig. 2c, respectively. 


\section{Temperature dependence of spin diffusion}

In order to study the spin dynamics we exploit the Hanle effect [1]. A set of the Hanle curves for different temperatures is shown in Fig. 4a. To avoid a contribution from the spin diffusion these curves were measured for a laser spot
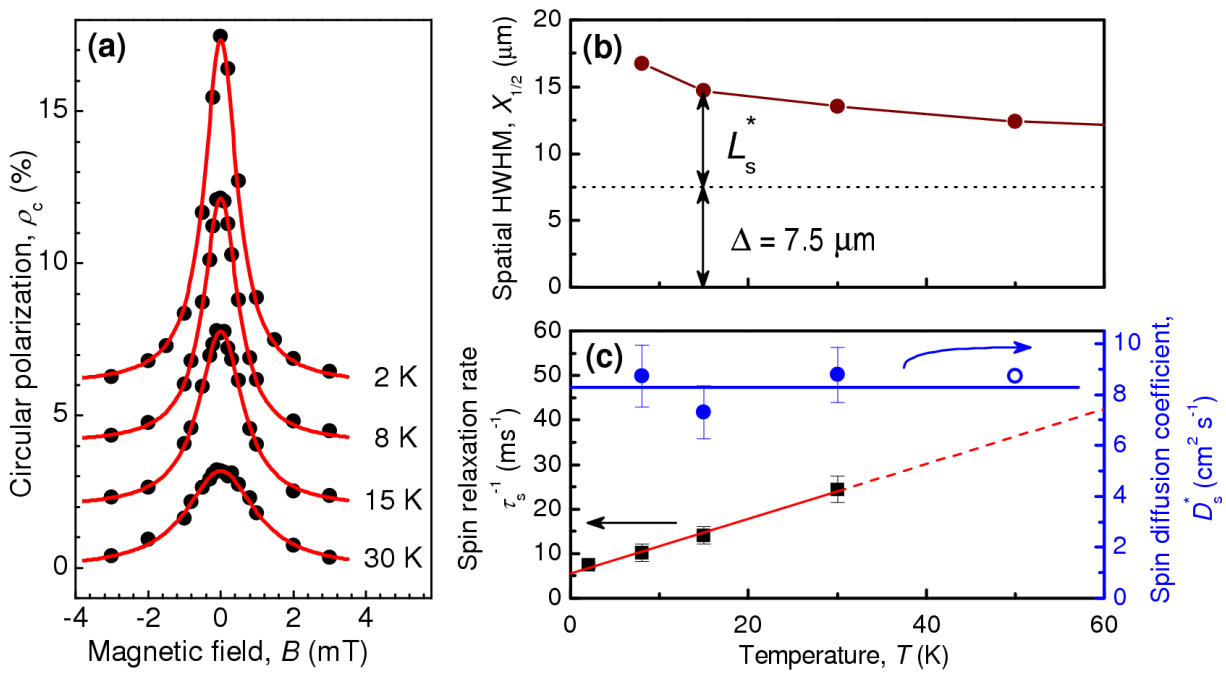

Fig. 4. Temperature dependence of spin relaxation and spin diffusion. (a) Hanle curves for different temperatures measured as the PL polarization, $\rho_{\mathrm{c}}$. Excitation intensity is $0.1 \mathrm{~kW} \mathrm{~cm}^{-2}$. The lines are Lorentzian fits [1]. The curves are shifted by $2 \mathrm{pp}$ for clarity. (b) Spatial spin profile half width $\left(X_{1 / 2}\right)$ vs. temperature. Arrows illustrate the estimate of the spin diffusion length $L_{\mathrm{s}}^{*}$. Pump intensity is $1.6 \mathrm{~kW} \mathrm{~cm}{ }^{-2}$, probe $0.1 \mathrm{~kW} \mathrm{~cm}^{-2}$. (c) Left axis: spin relaxation rate $\tau_{\mathrm{s}}^{-1}$ vs. temperature as found from the Hanle curves. The dashed line is a linear extrapolation. Right axis: spin diffusion coefficient $D_{\mathrm{s}}^{*}$ vs. temperature. Filled circles are experimental data, open circle is based on the extrapolation of $\tau_{\mathrm{s}}^{-1}$.

much larger $(c a .100 \mu \mathrm{m})$ than the spin diffusion length. The spin lifetime is extracted from the Lorentzian fit of these data. For each temperature this spin lifetime is extrapolated to zero pump power in order to avoid a contribution of photogenerated holes to the spin decay channel and obtain the spin relaxation time $\tau_{\mathrm{s}}$ of the undisturbed system. In particular, for $T=8 \mathrm{~K}$ we find $\tau_{\mathrm{s}}=98 \mathrm{~ns}$. The inverse of the resulting spin relaxation times is plotted as a function of temperature in Fig. 4c (left axis). The data is fitted well by a linear dependence enabling extrapolation to higher temperatures. Figure $4 \mathrm{~b}$ shows the spatial HWHM $\left(X_{1 / 2}\right)$ of the spin diffusion profile as a function of temperature. In order to remove the contribution of the finite pump and probe spot size to $X_{1 / 2}$ we consider the quantity $L_{\mathrm{s}}^{*}=X_{1 / 2}-\Delta$, from which one can calculate an effective spin diffusion 
coefficient $D_{\mathrm{s}}^{*}=\left(L_{\mathrm{s}}^{*}\right)^{2} \tau_{\mathrm{s}}^{-1}$ as function of temperature, as shown in Fig. 4c (right axis). The diffusion coefficient $D_{\mathrm{s}}^{*} \approx 8 \mathrm{~cm}^{2} \mathrm{~s}^{-1}$ appears to be a constant between 8 and $50 \mathrm{~K}$.

\section{Conclusions}

We demonstrated that the laser powers have a non-trivial influence on the spin transport in two-color Hanle-MOKE experiments. In particular, we found that the effective spin diffusion coefficient increases with pump power but remains constant with rising temperature. Especially strong focusing as required in spatially resolved experiments leads to rapid saturation of the spin polarization with growing pump intensity and provides an efficient spin decay channel with growing probe intensity.

\section{Acknowledgments}

We thank T. Kiessling for useful discussions. This research was supported by the DFG (SPP 1285).

\section{References}

[1] Optical Orientation, Eds. F. Meyer, B.P. Zakharchenya, North-Holland, Amsterdam 1984.

[2] R.I. Dzhioev, B.P. Zakharchenya, V.L. Korenev, M.N. Stepanova, Phys. Solid State 39, 1765 (1997).

[3] M. Oestreich, J. Hubner, D. Hagele, P.J. Klar, W. Heimbrodt, W.W. Ruhle, D.E. Ashenford, B. Lunn, Appl. Phys. Lett. 74, 1251 (1999).

[4] R. Fiederling, M. Keim, G. Reuscher, W. Ossau, G. Schmidt, A. Waag, L.W. Molenkamp, Nature 402, 787 (1999).

[5] Y. Ohno, D.K. Young, B. Beschoten, F. Matsukura, H. Ohno, D.D. Awschalom, Nature 402, 790 (1999).

[6] S.A. Crooker, D.L. Smith, Phys. Rev. Lett. 94, 236601 (2005).

[7] S.A. Crooker, M. Furis, X. Lou, C. Adelmann, D.L. Smith, C.J. Palmstrøm, P.A. Crowell, Science 309, 2191 (2005).

[8] S.A. Crooker, M. Furis, X. Lou, P.A. Crowell, D.L. Smith, C. Adelmann, C.J. Palmstrøm, J. Appl. Phys. 101, 081716 (2007).

[9] H. Hoffmann, G.V. Astakhov, T. Kiessling, W. Ossau, G. Karczewski, T. Wojtowicz, J. Kossut, L.W. Molenkamp, Phys. Rev. B 74, 073407 (2006). 